\title{
I WORKSHOP PORTUGAL E A ANTÁRCTIDA E A PREPARAÇÃO DAS ACTIVIDADES PORTUGUESAS NO ÂMBITO DO ANO POLAR INTERNACIONAL 2007-08
}

\author{
GONÇALO TELES VIEIRA ${ }^{1}$ \\ JOSÉ XAVIER ${ }^{2}$ \\ MÁRIO NEVEs ${ }^{3}$
}

A Antárctida é uma região chave no quadro das alterações climáticas e ambientais da Terra, pois contribui de modo sensível para a regulação do clima do planeta. O Oceano Antárctico alberga um valioso património biogenético e é rico em recursos marinhos, possuindo a região também importantes recursos minerais. Além disso, o continente contém quase $90 \%$ da água doce da Terra, aspecto suficiente para que a humanidade lhe preste especial atenção. Tendo em conta a importância da Antárctida e também do Árctico, o Conselho Internacional para a Ciência (ICSU) e a Organização Meteorológica Mundial (OMM) declararam o período de 1 de Março de 2007 a 1 de Março de 2009, como Ano Polar Internacional (API) (http://www.ipy.org). Neste momento, são já mais de quarenta as organizações governamentais e não-governamentais que apoiam ou subscrevem o API, sendo que a iniciativa conta actualmente com trinta e quatro comités ou contactos nacionais.

Tendo em conta este quadro global e a ausência de um interesse formal de Portugal pela Antárctida, organizou-se um workshop designado Portugal e a Antárctida. Oportunidades para a divulgação científica e para a investigação no âmbito do Ano Polar Internacional 2007-08, dedicado às actividades científicas e de divulgação nacionais relacionadas com a Antárctida. O evento, que juntou cerca de cento e vinte participantes na Faculdade de Letras de Lisboa, no dia 18 de Novembro de 2005, foi organizado pelo Centro de Estudos Geográficos da Universidade de Lisboa (CEG-UL), pelo Departamento de Geografia da mesma universidade e pelo Comité Nacional para o Ano Polar Internacional 2007-08. A Comissão Organizadora foi composta por Gonçalo Teles Vieira, Mário Neves e José Xavier. A iniciativa contou com o apoio da Faculdade de Letras da Universidade de Lisboa, da Associação de Estudantes da mesma faculdade e da Sociedade de Geografia de Lisboa.

O workshop juntou cientistas, exploradores, desportistas, divulgadores científicos, professores de vários graus de ensino, alunos, jornalistas, representantes de ONG e de

1 Professor Auxiliar da Faculdade de Letras da Universidade de Lisboa. Investigador do Centro de Estudos Geográficos. E-mail: gtvieira@ceg.ul.pt

2 Investigador do Centro de Ciências do Mar, Universidade do Algarve. E-mail: jxavier@ualg.pt

3 Professor Auxiliar da Faculdade de Letras da Universidade de Lisboa. Investigador do Centro de Estudos Geográficos. E-mail: marioneves@ceg.ul.pt 
associações profissionais e outros interessados nas regiões polares, e teve como objectivo dinamizar as actividades portuguesas a realizar no âmbito do API.

Os trabalhos desenvolveram-se em torno de várias palestras apresentadas por oradores convidados, distribuídas por quatro temas (Quadro I):

\section{Sessão 1 - A Antárctida e o Ano Polar Internacional}

A primeira palestra, proferida pelo Prof. Luís Mendes-Victor, Presidente do Comité Nacional para o Ano Polar Internacional, incidiu sobre a história dos anos polares anteriores, iniciada em 1882-83, com o segundo evento em 1932-33. Esta série culminou em 1957-58 no importantíssimo Ano Geofísico Internacional (no qual Mendes-Victor participou activamente), e que lançou as bases para o Tratado da Antárctida. O 4. ${ }^{\circ}$ evento, a ter lugar em 2007-08, constitui, por isso, a oportunidade ideal para Portugal desenvolver as suas actividades polares e estreitar as suas ligações históricas ao hemisfério sul.

A palestra do Comandante Agostinho Ramos da Silva, coordenador do Núcleo da Antárctida da Sociedade de Geografia de Lisboa, que participou na campanha Antárctida brasileira em 1991, versou a história da exploração da Antárctida, em especial a partir de finais do século XIX e princípios do século XX. De particular interesse, foram as referências à indústria baleeira e à caça às focas, actividades em que deverão ter participado vários portugueses, os quais precederam o desenvolvimento da actividade científica na Antárctida. José Nogueira, emigrante português no Chile, foi um exemplo do sucesso dos portugueses na caça aos lobos marinhos na segunda metade do século XIX, tendo prosperado e constituído uma das maiores fortunas de então na Terra do Fogo. Muito da história dos portugueses nas regiões polares está por estudar, considerando que a nossa influência se estendeu desde a Antárctida até ao arquipélago de Svalbard no Árctico. Este será, por certo, um tema fascinante e em aberto para comemorar o próximo API.

\section{Sessão 2 - Porquê a Antárctida? Uma visão dos cientistas portugueses que trabalham no continente gelado}

A sessão teve início com a palestra do Professor Gonçalo Teles Vieira, investigador que, desde 1999, desenvolve trabalhos sobre a dinâmica do permafrost (solo permanentemente gelado) e da camada activa nas Ilhas Shetlands do Sul (Península Antárctica). A apresentação incidiu sobre a importância da Antárctida para a regulação climática da Terra, em especial por se tratar de um foco de frio, que funciona como um verdadeiro refrigerador do Planeta. Para isto, as características da criosfera antárctica são de particular importância, destacando-se a calote glaciária de vários quilómetros de espessura e a sua dinâmica, as grandes plataformas de gelo e as superfícies de mar gelado (banquise), importantes componentes do balanço energético terrestre. Apesar de distante, a Antárctida é um elemento fundamental do sistema global, e as mudanças ambientais ocorridas na região terão consequências directas em todo o Mundo, e naturalmente, também em Portugal. Cabe, por isso, a todos os países envidar esforços para o estudo da Antárctida, e os estudos da criosfera são uma das áreas em que Portugal poderá contribuir mais fortemente.

A palestra do Doutor António Pedro Viterbo centrou-se nas características do clima da Antárctida, tendo sido focados os efeitos dinâmicos causados pelo continente, como 
a barreira ao fluxo zonal associada à extensão para norte da Península Antárctica, a influência da topografia glaciária na geração dos ventos catabáticos, e o papel da Antárctida no arrefecimento das águas oceânicas. Foi também analisada a influência do fenómeno El Niño no clima da Antárctida, e salientado o facto de a Antárctida constituir um laboratório do sistema climático, de extremo interesse para a análise da dinâmica atmosférica e oceânica. A contribuição de António Pedro Viterbo mostrou também como investigadores portugueses poderão colaborar no esforço de modelação do clima da Antárctida e, em particular, na integração dos resultados obtidos nos modelos de circulação global da atmosfera.

A palestra do Doutor José Xavier centrou-se na biologia da Antárctica, mostrando como através de estudos neste campo se podem testar hipóteses com relevância, quer à escala regional, quer mundial. Usando exemplos da sua colaboração com investigadores do British Antarctic Survey, José Xavier mostrou as ligações entre o aquecimento global e a oceanografia (e consequentemente na produção primária), na biodiversidade dos organismos marinhos e mesmo no comportamento de predadores do topo da cadeia alimentar. A conservação e gestão dos recursos marinhos foram também assuntos abordados, mostrando que a Antárctida poderá ser tomada como um exemplo de gestão racional dos recursos.

Para concluir a sessão, o Professor Adelino Canário apresentou uma caracterização da história evolutiva dos peixes da Antárctida, de que resultaram especializações anatómicas e fisiológicas, que os tornam extremamente vulneráveis a alterações ambientais. Depois de ter enunciado as principais etapas na evolução do continente, que culminaram na formação da barreira que constitui a Frente Polar, detalhou algumas especializações que foram adquiridas pela fauna piscícola - as particularidades da hemoglobina e o seu desaparecimento nalgumas espécies de peixes do gelo, compensações cardiovasculares para a perda de hemoglobina, a tendência para a redução do número de fibras musculares e para o aumento da densidade de mitocôndrias, uma intrigante diminuição da capacidade de resposta ao stress e o aparecimento de proteínas anticongelantes que inibem o crescimento de cristais de gelo. Finalmente, fez referência às especificidades do genoma das espécies piscícolas da Antárctida, ao seu interesse do ponto de vista científico e ao seu potencial biotecnológico.

\section{Sessão 3 - A Antárctida: continente de aventura}

A sessão dedicada à aventura visou integrar na iniciativa do API os aventureiros portugueses que têm praticado actividades nas regiões polares. Além do reconhecido mérito das viagens efectuadas, a relação entre ciência e sociedade só poderá sair fortalecida com o aprofundar desta relação. Efectivamente, além da possível colaboração em actividades científicas por parte dos exploradores polares, a visibilidade que as iniciativas de exploração e desportivas têm junto à juventude é merecedora da maior atenção por parte dos cientistas, e trará certamente muitas mais-valias na divulgação dos problemas das regiões polares. Aliás, como referiu a jornalista Margarida Pinto Correia, que se tem interessado pela temática da Antárctida e já a visitou, quem contacta com aquela região do globo, vê-se envolvido num meio natural tão puro e intocado, que inevitavelmente se tornará um embaixador do continente.

A palestra apresentada pelo Comandante José Inácio Jr. ilustrou a sua viagem de oito meses num pequeno veleiro até à Antárctida em 2001, dando particular relevo à espectacularidade ambiental da região, e em especial aos aspectos faunísticos. O entu- 
siasmo que tem acolhido a exposição da viagem de José Inácio Jr. em vários eventos públicos mostra o grande potencial que este tipo de trabalho tem na divulgação dos problemas ambientais do continente.

O Dr. Filipe Palma falou sobre a travessia a solo do Estreito de Gerlach (Península Antárctida), em caiaque, efectuada em Fevereiro de 2005. Esta aventura, marcada por grande espectacularidade, mostrou bem as dificuldades que o Homem tem em sobreviver nas condições antárcticas. Foi uma apresentação muito bem concebida e que aproximou a audiência dos problemas do dia-a-dia de uma aventura como a efectuada, tendo sido explicados os problemas de organização e logística, bem como os acontecimentos ocorridos durante aquela semana de isolamento. Filipe Palma é também um bom exemplo de divulgação dos problemas do continente, pois tem efectuado várias conferências, exposições e visitas a escolas.

\section{Sessão 4 - 0 Ano Polar Internacional: uma oportunidade para a divulgação cientí- fica e para a cultura}

Esta sessão pretendeu apresentar experiências nacionais de divulgação da região antárctica, tendo-se convidado, para isso, a Dra. Patrícia Filipe, coordenadora área da educação do Oceanário de Lisboa, e os Arquitectos Bernardo Nogueira Vaz e Diogo Teixeira da Expoente - Iniciativas Artísticas e Culturais.

A palestra da Dra. Patrícia Filipe versou as actividades do Oceanário de Lisboa e o modo como esta instituição tem contribuído para divulgar os problemas dos oceanos, e como tem incluído na sua agenda o Oceano Antárctico. O Oceanário, desde o início, tem apoiado o API perspectivando-se várias colaborações no âmbito do evento, que certamente irão contribuir decisivamente para o alargar dos públicos alvo desta iniciativa internacional.

A Expoente - Iniciativas Artísticas e Culturais trouxe ao workshop o filme Noite em branco, que conta a experiência antárctica de Paulo Afonso (direcção científica), Olivier Blanc (realização, imagem e som) e de António Marques (fotografia), que participaram em 2001 na campanha antárctica do navio alemão Polarstern. Trata-se de uma obra de autor, com banda sonora de Bernardo Sassetti e que convida à reflexão, ilustrando de forma exemplar o modo como decorre a actividade científica num navio oceanográfico polar.

O evento contou ainda com uma sessão de posters de grupos de investigação portugueses com trabalhos na Antárctida (Quadro II). Além do Centro de Estudos Geográficos e do Centro de Geofísica da Universidade de Lisboa, e do Centro de Ciências do Mar da Universidade do Algarve, atrás mencionados, estiveram representados o Centro de Geofísica da Universidade de Évora, o Departamento de Oceanografia e Pescas da Universidade dos Açores, a Escola Superior Agrária do Instituto Politécnico de Coimbra, o Instituto Geofísico da Universidade de Coimbra e a Unidade de Investigação em EcoEtologia do Instituto Superior de Psicologia Aplicada. O evento contou, assim, com a presença de representantes de oito grupos de investigação portugueses, o que mostra que existe um interesse nacional significativo na investigação na Antárctida. Por colaborarem com estes grupos, estiveram também representados sete centros de investigação estrangeiros: o British Antarctic Survey, o Centre National de la Recherche Scientifique (C.E.F.E. e C.E.B.C), o Departamento de Geografia da Universidade de Zurique, o Departamento de Zoologia da Universidade de Cambridge, o Grupo de Física Ambiental da 
Universidade de Alcalá de Henares e o Instituto de Ciências da Atmosfera e do Clima de Bolonha (ISAC-CNR).

O workshop culminou com o fórum Portugal e o Ano Polar Internacional: ciência e sociedade num continente de fascínio, onde foram apresentados os objectivos do API, e discutidas formas de Portugal contribuir para o evento. Os principais objectivos traçados pelos membros do Comité Nacional para o API são: a assinatura por parte de Portugal do Tratado da Antárctida; o desenvolvimento das actividades científicas nacionais nas regiões polares; a adesão ao SCAR - Scientific Committee for Antarctic Research (organismo que coordena a investigação científica na Antárctida); e a organização de diversas actividades ligadas à educação, divulgação e promoção da importância da Antárctida para o equilíbrio do Sistema Terra. Estes objectivos foram discutidos no fórum, enquadrados em três grandes temas: a) Divulgação científica e educação; b) Como fazer ciência na Antárctida? e c) Antárctida fonte de inspiração artística. A elevada participação no fórum permitiu a discussão de várias propostas de actividades a realizar no API, sendo de destacar, em particular no que respeita à educação e divulgação, o interesse na colaboração por parte do Oceanário de Lisboa (que, aliás, deu importante apoio na divulgação do workshop), Fundação do Gil, Sociedade de Geografia de Lisboa, Associação Portuguesa de Geógrafos, Associação de Professores de Geografia, Associação Portuguesa para a Dinamização do Mergulho, Expoente - Iniciativas Artísticas e Culturais e Viagens Fim do Mundo.

Aproveitando o entusiasmo e a grande dinâmica criada em torno do workshop, a Comissão Organizadora irá contactar todos os participantes, convidando-os a integrar a mailing list API-PT (http://br.groups.yahoo.com/group/api-pt), de forma a dar seguimento aos trabalhos iniciados. Será ainda feita uma sistematização das ideias apresentadas e disponibilizado no site português do API (http://anopolar.no.sapo.pt), um formulário para envio de propostas, que constituirão uma base de dados on-line usada para organizar e divulgar as iniciativas em torno do Ano Polar Internacional 2007-08.

Este primeiro workshop foi um importante passo para planear as actividades portuguesas do API. A dinâmica demonstrada pelos participantes abre excelentes perspectivas quanto aos desenvolvimentos futuros da investigação científica nacional nas regiões polares e, em particular, na Antárctida. A qualidade e número de trabalhos científicos apresentados demonstra que existe em Portugal massa crítica suficiente para criar uma actividade continuada de investigação na Antárctida, que poderá contribuir com eficácia para o esforço científico internacional, actualmente conduzido naquela região, e que se pretende estimular durante e a partir do API. Estamos seguros de que o workshop constituiu, pois, um ponto de partida e um arranque para outras iniciativas, e que as ideias discutidas serão levadas a bom termo de forma a que o Ano Polar Internacional 2007-08 possa ser um acontecimento com sucesso garantido no nosso país. 
Quadro I - Palestras apresentadas no I Workshop Portugal e a Antárctida Table I - Lectures presented in the I Workshop Portugal and Antarctida

\section{SESSÃO 1 - A ANTÁRCTIDA E O ANO POLAR INTERNACIONAL 2007-08}

O Ano Polar Internacional 2007-08: um marco na investigação científica internacional e uma oportunidade para Portugal.

Prof. Doutor Luís Mendes-Victor, Presidente do Comité Português para o Ano Polar Internacional, Centro de Geofísica, Universidade de Lisboa

A descoberta da Antárctida

Com. Agostinho Ramos da Silva, Sociedade de Geografia de Lisboa

\section{SESSÃO 2 - PORQUÊ A ANTÁRCTIDA? UMA VISÃO DOS CIENTISTAS PORTUGUESES QUE TRABALHAM NO CONTINENTE GELADO}

A Criosfera Antárctica e a sua importância para o Sistema Terra

Prof. Doutor Gonçalo Vieira, Centro de Estudos Geográficos, Universidade de Lisboa

Clima e Variabilidade Climática da Antárctida

Doutor António Pedro Viterbo, Instituto de Meteorologia

A Biologia do Oceano Antárctico e aplicações práticas na investigação dos recursos marinhos

Doutor José Xavier, Centro de Ciências do Mar, Universidade do Algarve

Ecofisiologia e Genomas na Antárctida e sua importância global

Prof. Doutor Adelino Canário, Centro de Ciências do Mar, Universidade do Algarve

\section{SESSÃO 3 - A ANTÁRCTIDA: CONTINENTE DE AVENTURA}

De veleiro até à Antárctida

Com. José Inácio Jr.

A travessia do Estreito de Gerlach em caiaque

Dr. Filipe Palma

\section{SESSÃO 4 - O ANO POLAR INTERNACIONAL: UMA OPORTUNIDADE PARA A DIVULGAÇÃO CIENTÍFICA E PARA A CULTURA}

A Antárctida na agenda do Oceanário de Lisboa - sensibilização para a conservação dos oceanos Dra. Patrícia Filipe, Oceanário de Lisboa

Filme: Noite em Branco

Expoente-Actividades Artísticas e Culturais 
Quadro II - Posters de grupos de investigação portugueses apresentados no Workshop Portugal e a Antárctida.

Table II - Posters made by portuguese research groups and presented in the I Workshop Portugal and Antarctida

Stratospheric nitrogen dioxide and ozone measurements in Antarctica - current activities and future perspectives

Daniele Bortoli ${ }^{1,2}$, G. Giovanelli² e Ana Maria Silva ${ }^{1}$

${ }^{1}$ Geophysics Centre of Évora, University of Évora, Portugal; ${ }^{2}$ Institute of Atmospheric Sciences and Climate

(ISAC-CNR), Bologna, Itália.

Considerações comparativas sobre o arrefecimento do Antárctico, registado durante 1986-2000

Ryunosuke Kikuchi ${ }^{1}$, S. Santos ${ }^{1}$ e R. Gerardo ${ }^{2}$

${ }^{1}$ CERNAS, ESAC - Instituto Politécnico de Coimbra, ${ }^{2}$ Dep. CEA, ESAC-Instituto Politécnico de Coimbra.

Environmental controls on the spatial distribution of periglacial landforms: a case-study from Hurd Peninsula, Livingston Island, Antarctic

Gonçalo Vieira ${ }^{1}$ and Miguel Ramos ${ }^{2}$

${ }^{1}$ Centro de Estudos Geográficos, University of Lisbon, Portugal; , ${ }^{2}$ Department of Physics, University of Alcalá de Henares, Spain.

Permafrost and active layer monitoring and modelling in Livingston and Deception Islands (South Shetlands, Antarctic) - PERMAMODEL

Miguel Ramos ${ }^{1}$, Gonçalo Vieira², Jose Agustín Garcia ${ }^{3}$, Martin Hoelzle ${ }^{4}$ and Stephan Gruber ${ }^{4}$

${ }^{1}$ Department of Physics, University of Alcalá de Henares, Spain; ${ }^{2}$ Centro de Estudos Geográficos, University of Lisbon, Portugal; ${ }^{3}$ Department of Physics, University of Extremadura, Badajoz, Spain; ${ }^{4}$ Department of Geography, University of Zurich, Switzerland.

À caça de meteoritos no Planalto Antárctico

Vera Assis Fernandes

Instituto Geofísico, Universidade de Coimbra, Portugal.

Um recorde de velocidade, à boleia de uma tempestade antárctica

Paulo Catry ${ }^{1,2}$, Richard A. Phillips ${ }^{2}$ and John P. Croxall ${ }^{2}$

${ }^{1}$ Unidade de Investigação em Eco-Etologia, ISPA, Lisboa; ${ }^{2}$ British Antarctic Survey, Natural Environment Research Council, Cambridge, UK.

Can albatrosses be cephalopod dependent?

José C. Xavier ${ }^{1,2,3}$, John P. Croxall ${ }^{2}$, Paul G. Rodhouse ${ }^{2}$, Keith Reid ${ }^{2}$ and Phil. N. Trathan ${ }^{2}$

${ }^{1}$ University of Cambridge, Department of Zoology, Cambridge, UK; ${ }^{2}$ British Antarctic Survey, Natural Environment Research Council, Biosciences Division, Cambridge, UK; ${ }^{3}$ Centre of Marine Sciences (CCMAR), Faculty of Marine Sciences and Environment, University of Algarve, Faro, Portugal.

Insular bird populations can be saved from rats: a long term experimental study of White-chinned Petrels Procellaria aequinoctialis in the Crozet archipelago

Joël Bried ${ }^{1,2}$, Pierre Jouventin ${ }^{1}$ and Thierry Micol ${ }^{3,4}$

${ }^{1}$ C.N.R.S., C.E.F.E., Montpellier France; ${ }^{2}$ Departamento de Oceanografia e Pescas, Centro do IMAR da Universidade dos Açores, Horta, Faial, Açores, Portugal; ${ }^{3}$ C.N.R.S., C.E.B.C., Beauvoir sur Niort, France; ${ }^{4}$ T.A.A.F., Saint Pierre, La Réunion. 